Quim. Nova, Vol. 34, No. 5, 874-878, 2011

\title{
IDENTIFICAÇÃO DO CARBAMATO DE ETILA DURANTE O ARMAZENAMENTO DA CACHAÇA EM TONEL DE CARVALHO (Quercus sp) E RECIPIENTE DE VIDRO
}

\author{
Jeancarlo Pereira dos Anjos, Maria das Graças Cardoso*, Adelir Aparecida Saczk, Lidiany Mendonça Zacaroni e Wilder \\ Douglas Santiago \\ Departamento de Química, Universidade Federal de Lavras, CP 3037, 37200-000 Lavras - MG, Brasil \\ Haroldo Silveira Dórea \\ Departamento de Química, Universidade Federal de Sergipe, Av. Marechal Rondon, s/n, 40100-000 São Cristovão - SE, Brasil \\ Ana Maria de Resende Machado \\ Departamento de Ciência dos Alimentos, Universidade Federal de Lavras, CP 3037, 37200-000 Lavras - MG, Brasil
}

Recebido em 30/7/10; aceito em 16/11/10; publicado na web em 25/2/11

\begin{abstract}
IDENTIFICATION OF ETHYL CARBAMATE DURING THE AGING OF CACHAÇA IN AN OAK BARREL (Quercus sp) AND A GLASS VESSEL. The objective of the present study was the identification and quantification of ethyl carbamate (EC), by HPLC-FLD, after different periods of storage in an oak (Quercus sp) barrel and a glass vessel. The concentration of EC in the cachaça samples varied from $<\mathrm{LD}$ to $17.46 \mu \mathrm{g} \mathrm{L} \mathrm{L}^{-1}$, for the period during which the cachaça was stored in an oak barrel, and <LQ to $18.51 \mu \mathrm{g} \mathrm{L}{ }^{-1}$ for the cachaça stored in a glass recipient. The storage of the beverage in both the oak barrel and the glass vessel resulted in a significant increase in the concentration of this contaminant.
\end{abstract}

Keywords: cachaça; ethyl carbamate; HPLC-FLD.

\section{INTRODUÇÃO}

A cachaça é uma bebida tradicionalmente produzida no Brasil, obtida a partir do mosto fermentado de cana-de-açúcar. Pelo fato de apresentar diferenças na sua composição química, o estudo de compostos potencialmente tóxicos, considerados como contaminantes, tem sido um fator determinante no controle de qualidade da bebida. ${ }^{1}$

Dentre estes contaminantes o carbamato de etila, composto potencialmente carcinogênico, tem sido alvo de inúmeras pesquisas pelo fato de ser encontrado em diversos alimentos e bebidas fermentadas, e por ainda não estar bem esclarecida sua origem durante o processo de obtenção da cachaça, apesar de que diferentes meios têm sido propostos para a formação deste contaminante. ${ }^{2-4}$ Mesmo em pequenas quantidades, este é um composto tóxico ao homem. A legislação brasileira estabeleceu um limite máximo de $150,0 \mu \mathrm{g} \mathrm{L}^{-1}$ para esta substância em aguardentes, ${ }^{5}$ baseando-se na legislação do Canadá que há algum tempo tem demonstrado preocupação quanto à presença desta em alimentos e bebidas.

O carbamato de etila (CE), também conhecido como uretana, é o éster do ácido carbâmico e apresenta a fórmula molecular $\mathrm{C}_{3} \mathrm{H}_{7} \mathrm{O}_{2} \mathrm{~N}$. ${ }^{6}$ É formado naturalmente durante processos fermentativos, podendo ser encontrado em diversos alimentos e bebidas tais como pão, iogurte, vinho, cerveja e, principalmente, em bebidas fermento-destiladas como uísque, rum, vodca, grapa, cachaça e tiquira. ${ }^{7}$

Bruno et $a l .{ }^{2}$ e Polastro et al. ${ }^{8}$ citam que o principal mecanismo proposto para a formação do CE ocorre por meio do metabolismo das leveduras durante o processo fermentativo, onde há a degradação enzimática da arginina em ureia, a qual reage com etanol produzindo o CE. Além da presença de precursores nitrogenados durante o processo de destilação e de altas temperaturas, sob condições ácidas, Iida et al. ${ }^{9}$ citam que esta reação é favorecida quando se utiliza longo

\footnotetext{
*e-mail: mcardoso@dqi.ufla.br
}

período de armazenamento do produto. Outras reações relevantes que promovem a formação do CE em bebidas alcoólicas envolvem reações entre o etanol e diferentes precursores nitrogenados, tais como fosfato de carbamila e cianeto. ${ }^{6}$ Masson ${ }^{10}$ menciona que este último é considerado um precursor durante e após o processo de destilação.

O método considerado como oficial para a análise de CE em bebidas alcoólicas envolve a utilização da cromatografia gasosa acoplada à espectrometria de massas (GC/MS) após a execução de técnicas de preparação de amostras (etapas de extração e/ou clean-up) devido às baixas concentrações deste composto em bebidas. ${ }^{11}$ Porém, alguns autores têm utilizado a cromatografia líquida de alta eficiência com detector de fluorescência (HPLC-FLD) para a determinação de CE em diferentes matrizes. Este método tem se apresentado como uma alternativa à substituição ao método oficial, pois apresenta níveis de detecção similares além de proporcionar maior rapidez na etapa de preparação das amostras, já que não necessita de etapas prévias de extração e concentração do analito. Este método envolve, como preparação da amostra, apenas a derivação prévia do CE (Figura 1) permitindo, assim, sua detecção utilizando o detector de fluorescência. ${ }^{11,12}$<smiles>CCOC(=O)NC1c2ccccc2Oc2ccc(Cl)cc21</smiles>

$$
\text { 9-xantidrol }
$$

carbamato de etila

Figura 1. Reação de derivação do carbamato de etila utilizando como agente derivatizante 9-xantidrol, sob condições ácidas

Diante do exposto, este trabalho teve como objetivos identificar e quantificar o carbamato de etila, utilizando a cromatografia líquida 
de alta eficiência com detector de fluorescência (HPLC-FLD), em diferentes períodos de armazenamento da cachaça em tonel de carvalho (Quercus sp) e recipiente de vidro.

\section{PARTE EXPERIMENTAL}

\section{Obtenção das amostras}

A cachaça utilizada foi produzida em uma unidade produtora situada no município de Perdões/MG, no período da safra de 2008. Após o processo de destilação do vinho, foi coletada parte das frações "cabeça", "coração" (denominada de cachaça nova) e "cauda" do destilado.

Para o envelhecimento da bebida, o produto correspondente ao destilado do "coração" foi armazenado em tonel de madeira. O tonel foi mantido em galpão fechado com temperatura e umidade do ar não controlados, e colocado na posição horizontal para possibilitar o maior contato da bebida com a madeira e distanciado de outros toneis para possibilitar as trocas gasosas. Nesta etapa, foi armazenado um volume aproximado de $100 \mathrm{~L}$ da cachaça em um tonel de carvalho (Quercus sp), com capacidade de $200 \mathrm{~L}$.

Após armazenar a bebida em tonel de madeira, alíquotas desta foram coletadas a cada mês por um período de 12 meses, para o acompanhamento da concentração de CE na bebida. Conforme a legislação, este corresponde ao período mínimo em que a bebida deve ser armazenada em recipientes de madeira para ser considerada envelhecida. ${ }^{5}$ Foram consideradas, além das amostras coletadas ao longo do período de envelhecimento da bebida em tonel de carvalho, alíquotas da cachaça armazenada em recipiente de vidro ao longo dos 12 meses, a fim de simular o tempo de maturação da cachaça.

Até a data de realização das respectivas análises, todo o material coletado foi mantido sob refrigeração.

\section{Reagentes e padrões}

O método utilizado para a quantificação do CE consistiu na derivação prévia das amostras para posterior análise por cromatografia líquida de alta eficiência. ${ }^{12-14}$ Os reagentes empregados para análise foram padrão de carbamato de etila (Acros Organics), etanol, propanol, hexano, ácido clorídrico $(\mathrm{HCl})$, acetato de etila, acetato de sódio, acetonitrila grau HPLC (Merck), água ultrapura e 9-xantidrol (Acros Organics). Para as análises, foi realizada a purificação do 9-xantidrol por meio de sucessivas recristalizações em hexano.

\section{Derivação do padrão de carbamato de etila}

A reação química envolvida na etapa de derivação do CE está apresentada na Figura 1.

Em frasco âmbar, preparou-se uma solução de 9-xantidrol 0,2 mol $\mathrm{L}^{-1}$ em propanol. A esta, foram adicionados $20 \mathrm{~mL}$ de uma solução padrão de carbamato de etila $4,0 \mathrm{~g} \mathrm{~L}^{-1}$, preparada em etanol $40 \%$. Em seguida, adicionaram-se $2 \mathrm{~mL}$ de $\mathrm{HCl} 15 \mathrm{~mol} \mathrm{~L}^{-1} \mathrm{e}$, então, a mistura reacional foi mantida sob agitação por aproximadamente $1 \mathrm{~min}$. Após agitação, esta foi mantida em repouso por $60 \mathrm{~min}$. Os cristais obtidos foram filtrados e recristalizados em hexano.

Para a análise quantitativa, preparou-se uma solução estoque do carbamato de etila derivado numa concentração de $10 \mathrm{mg} \mathrm{L}^{-1}$, em acetato de etila. Para a construção da curva analítica, foram realizadas diluições em etanol $50 \%$ a partir da solução estoque previamente preparada, sendo preparadas soluções de trabalho em concentrações que variaram de 5,0 a 75,0 $\mu \mathrm{g} \mathrm{L}^{-1}$. A curva analítica continha seis pontos e a linearidade foi verificada por meio do método de regressão linear.

\section{Derivação das amostras}

Em frasco âmbar, adicionaram-se 4,0 mL de cachaça seguidos de $0,8 \mathrm{~mL}$ de solução de xantidrol $0,02 \mathrm{~mol} \mathrm{~L}^{-1}$ (preparada em propanol). Após agitação, adicionaram-se $0,4 \mathrm{~mL}$ de $\mathrm{HCl} 1,5 \mathrm{~mol} \mathrm{~L}^{-1}$, mantendo a mistura reacional sob agitação por $1 \mathrm{~min}$. Em seguida, esta foi mantida em repouso por $60 \mathrm{~min}$ sendo, posteriormente, filtrada em membranas de polietileno $0,45 \mu \mathrm{m}$ (Millipore).

\section{Condições cromatográficas e quantificação do carbamato de etila}

As análises do CE foram realizadas em um cromatógrafo líquido de alta eficiência Shimadzu, equipado com duas bombas de alta pressão modelo LC-6AD, um detector de fluorescência (FLD) modelo RF-10AXL, degaseificador modelo DGU-20A 3 , interface modelo CBM-20A e injetor automático com autoamostrador modelo SIL10AF. As separações foram realizadas empregando-se uma coluna Agilent - Zorbax Eclipse AAA (4,6 x 150 mm, 5 $\mu \mathrm{m})$ conectada a uma pré-coluna Agilent - Zorbax Eclipse AAA 4-Pack (4,6 x 12,5 $\mathrm{mm}, 5 \mu \mathrm{m})$.

A quantificação do CE foi realizada utilizando-se o método de padronização externa. Os comprimentos de onda de excitação e emissão empregados foram 233 e $600 \mathrm{~nm}$, respectivamente. O fluxo utilizado em toda a análise foi de $0,75 \mathrm{~mL} \mathrm{~min}^{-1}$ e o volume injetado das amostras e do padrão foi de $20 \mu \mathrm{L}$. A eluição foi realizada em sistema do tipo gradiente: 0 a 5 min (40-60\% B); 5 a 10 min $(60-70 \%$ B); 10 a $18 \min (70-80 \%$ B); 18 a $19,5 \min (80-90 \%$ B); 19,5 a 25 $\min (90-40 \%$ B); 25 a $30 \min (40 \%$ B). A fase móvel foi composta por solução de acetato de sódio $20 \mathrm{mM}$ (Solvente A) e acetonitrila (Solvente B).

O tempo de retenção médio obtido para o CE foi de $13,1 \pm 0,1$ min. A quantificação do CE nas amostras de cachaça foi realizada por meio da construção de uma curva analítica obtida por regressão linear $(\mathrm{y}=7112,82726 \mathrm{x}+40192,22186$; onde, $\mathrm{y}=$ área do pico e $\mathrm{x}=$ concentração de $\mathrm{CE}$ ), correlacionando a área do pico versus a concentração da respectiva solução padrão, sendo que o coeficiente de determinação $\left(\mathrm{r}^{2}\right)$ obtido foi de 0,9981 . Os limites de detecção (LD) e quantificação (LQ) foram estimados por meio dos parâmetros obtidos para a curva analítica construída, sendo calculados pelas respectivas relações matemáticas: $\mathrm{LD}=3 \mathrm{DP} / \mathrm{m}$ e $\mathrm{LQ}=10 \mathrm{DP} / \mathrm{m}$ (onde, $\mathrm{DP}=$ estimativa do desvio padrão da linha de regressão e $\mathrm{m}=$ coeficiente angular da linha de calibração). ${ }^{15}$ Para estes, foram encontrados os valores de 3,93 e 13,09 $\mu \mathrm{g} \mathrm{L}^{-1}$, respectivamente. Dados mais detalhados, relativos à validação do método proposto para a determinação de CE em cachaça, podem ser verificados nos trabalhos de Machado, ${ }^{16}$ que realizou a validação e comparação de diferentes métodos para a análise deste contaminante na bebida sendo, este, uma aplicação do método já validado pelo respectivo autor.

Todas as amostras foram analisadas em duplicata, enquanto as soluções de trabalho do padrão de carbamato de etila derivado foram analisadas em triplicata.

\section{Análise estatística}

Os dados obtidos foram submetidos à análise de variância, sendo as médias de cada modo de armazenamento da bebida comparadas pelo teste de Scott-Knott ao nível de 95\% de confiança, utilizando o programa estatístico Sisvar. ${ }^{17}$

\section{RESULTADOS E DISCUSSÃO}

A Figura 2 mostra o cromatograma obtido para o padrão de carbamato de etila, na concentração de $75,0 \mu \mathrm{g} \mathrm{L}^{-1}$. 


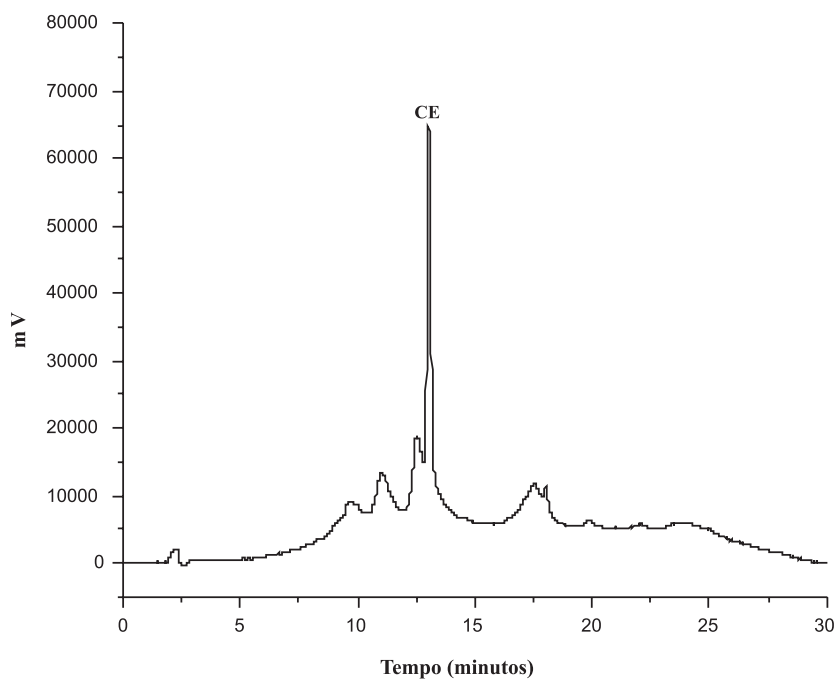

Figura 2. Cromatograma de uma solução padrão de carbamato de etila (CE) $\left(75,0 \mu g L^{-1}\right)$

Para garantir a reação completa do carbamato de etila, na derivação utiliza-se excesso de 9-xantidrol. Apesar do produto resultante da derivação também ter sido purificado, observa-se a presença de outros picos no cromatograma da Figura 2, conforme citado por Madrera e Valles, ${ }^{12}$ já que existe semelhança estrutural entre o 9-xantidrol e o derivado de carbamato, dificultando a purificação do analito derivatizado.

Os cromatogramas obtidos para as amostras de cachaça nova (cachaça não armazenada em tonel de madeira ou recipiente de vidro), para a cachaça após 12 meses de armazenamento no tonel de carvalho e para a cachaça após 12 meses de armazenamento em recipiente de vidro estão apresentados na Figura 3.

O LD e o LQ obtidos utilizando o método proposto estão dentro das respectivas faixas de concentração encontradas em trabalhos desenvolvidos recentemente referentes à determinação de $\mathrm{CE}$ em diferentes matrizes, utilizando a técnica de cromatografia líquida de alta eficiência com detecção de fluorescência (HPLC-FLD), com derivação prévia do carbamato de etila. Alguns trabalhos realizados com diferentes bebidas alcoólicas têm apresentado valores que variam de 1,64 a 4,2 $\mu \mathrm{g} \mathrm{L}^{-1}$, para o limite de detecção, e de 3,56 $\mu \mathrm{g} \mathrm{L}^{-1}$ a $4 \mathrm{mg} \mathrm{L}^{-1}$, para o limite de quantificação. $6,12,13$

A Tabela 1 mostra os resultados obtidos para a quantificação do CE nas frações "cabeça", "coração" (cachaça nova) e "calda".

Por meio dos resultados obtidos pode-se constatar que este contaminante se apresentou abaixo do limite de detecção para as respectivas frações. Trabalhos de Andrade Sobrinho et al. ${ }^{4}$ mostraram que as maiores concentrações de CE são encontradas na fração "cabeça", seguido das frações "coração" e "cauda", devido ao fato do CE ser mais solúvel em etanol $\left(1,2 \mathrm{~g} \mathrm{~mL}^{-1}\right)$ que em água $\left(0,10 \mathrm{~g} \mathrm{~mL}^{-1}\right)$. Por este fato, torna-se previsível que a "cabeça" apresente uma maior concentração de $\mathrm{CE}$, já que esta apresenta uma maior graduação alcoólica. Foram obtidos valores de 51,34; 44,41 e 23,27\% v/v para a graduação alcoólica das frações "cabeça", "coração" e "cauda", respectivamente, tendendo a reduzir o teor alcoólico da bebida ao longo do processo de destilação. Portanto, a separação eficiente das frações durante a destilação da cachaça de alambique pode ser uma alternativa na redução dos níveis de CE no destilado.

Para alguns autores, a realização da destilação da cachaça em alambiques de cobre poderia ser um agravante na formação do carbamato de etila, já que se acredita na possibilidade deste metal atuar como catalisador nas reações de formação deste contaminante. ${ }^{18}$ Porém, alguns estudos realizados recentemente, como os de Mas-
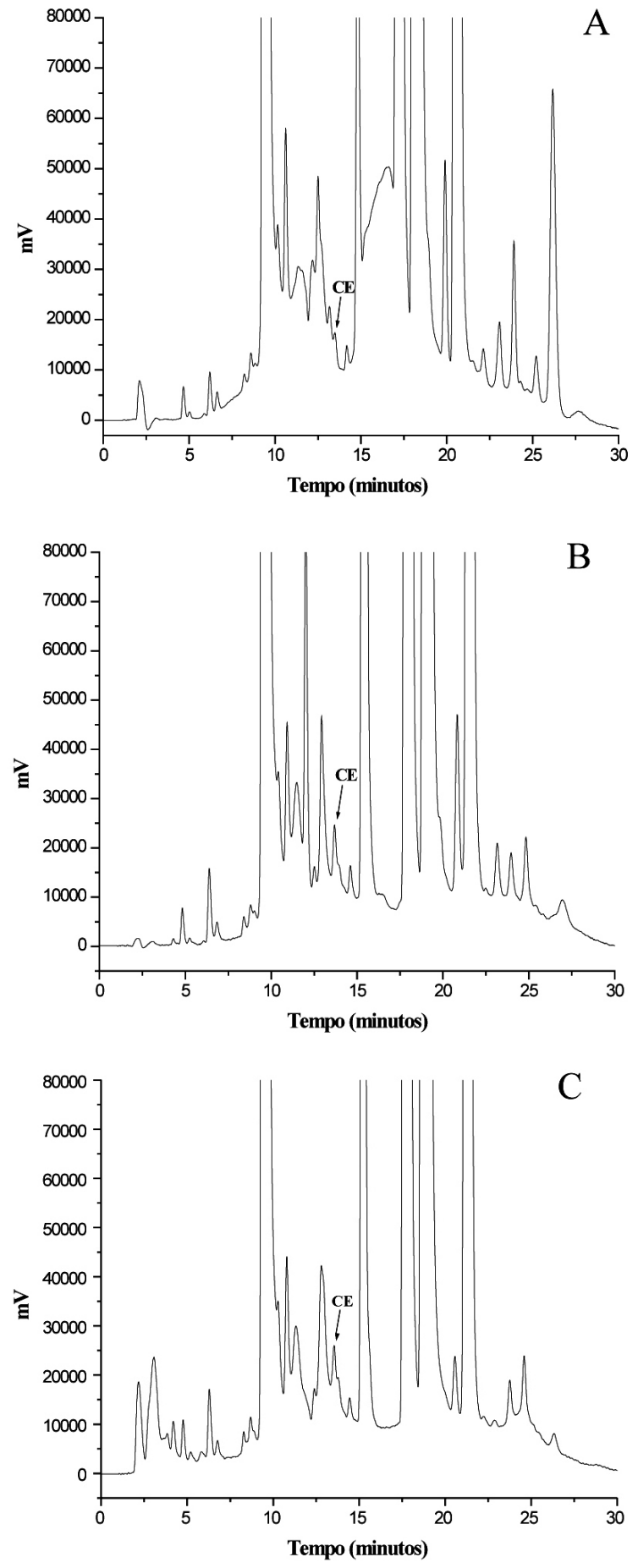

Figura 3. Cromatograma da amostra de cachaça nova (A), amostra de cachaça após 12 meses de armazenamento em tonel de carvalho $(B)$ e amostra de cachaça após 12 meses de armazenamento em recipiente de vidro $(C)$, com a identificação do pico do carbamato de etila (CE)

Tabela 1. Concentração de carbamato de etila nas frações "cabeça", "coração" (cachaça nova) e "cauda"

\begin{tabular}{cc}
\hline Fração da destilação & Carbamato de etila $\left(\mu \mathrm{g} \mathrm{L}^{-1}\right) *$ \\
\hline "Cabeça" & $<\mathrm{LD}$ \\
"Coração" (cachaça nova) & $<\mathrm{LD}$ \\
"Cauda" & $<\mathrm{LD}$ \\
\hline
\end{tabular}

$*<\mathrm{LD}=$ menor que limite de detecção 
son, ${ }^{10}$ Barcelos et al. ${ }^{19}$ e Zacaroni, ${ }^{20}$ demonstraram que não houve correlação entre as concentrações de cobre e carbamato de etila em cachaça. Portanto, devem ser investigados outros fatores e etapas do processo de produção que podem influenciar na formação do CE na bebida, já que a presença de cobre não se mostrou relevante para a formação deste.

Com o intuito de avaliar a influência da etapa de envelhecimento da cachaça na formação do CE, realizou-se a quantificação deste contaminante durante o período de envelhecimento da cachaça, em tonel de carvalho, e durante o período de armazenamento da mesma em recipiente de vidro (Tabela 2 ).

Tabela 2. Concentração de carbamato de etila durante o envelhecimento da cachaça em tonel de carvalho e durante seu armazenamento em recipiente de vidro

\begin{tabular}{ccc}
\hline $\begin{array}{c}\text { Tempo de armazenamento } \\
(\text { meses })\end{array}$ & \multicolumn{2}{c}{ Carbamato de etila $\left(\mu \mathrm{g} \mathrm{L}^{-1}\right)^{*}$} \\
Tonel de madeira & Vidro \\
\hline 1 & $<\mathrm{LD}$ & $<\mathrm{LQ}$ \\
2 & $<\mathrm{LQ}$ & $13,63 \pm 0,07$ \\
3 & $<\mathrm{LQ}$ & $15,67 \pm 0,13$ \\
4 & $<\mathrm{LQ}$ & $16,24 \pm 1,27$ \\
5 & $<\mathrm{LQ}$ & $17,01 \pm 0,05$ \\
6 & $<\mathrm{LQ}$ & $17,49 \pm 0,75$ \\
7 & $<\mathrm{LQ}$ & $18,37 \pm 0,21$ \\
8 & $<\mathrm{LQ}$ & $19,11 \pm 0,13$ \\
9 & $13,81 \pm 0,59$ & $17,60 \pm 0,91$ \\
10 & $14,53 \pm 0,74$ & $15,27 \pm 2,91$ \\
11 & $<\mathrm{LQ}$ & $17,44 \pm 0,00$ \\
12 & $17,46 \pm 1,60$ & $18,51 \pm 0,37$ \\
Média geral & 10,81 a1 & $16,65 \mathrm{a} 2$ \\
\hline
\end{tabular}

*Média \pm desvio padrão; médias seguidas de mesma letra e número são consideradas iguais pelo teste de Scott-Knott $(\alpha=5 \%)$

Por meio dos resultados obtidos pode-se constatar que a concentração de $\mathrm{CE}$ nas amostras de cachaça analisadas variou de $<\mathrm{LD}$ a $17,46 \mu \mathrm{g} \mathrm{L}^{-1}$, para o acompanhamento realizado com a cachaça armazenada em tonel de carvalho, e de $<\mathrm{LQ}$ a $18,51 \mu \mathrm{g} \mathrm{L}^{-1}$ para a cachaça armazenada em recipiente de vidro. Acredita-se que a falta de uma homogeneização eficiente no momento das coletas das alíquotas da bebida armazenada em tonel de carvalho (devido ao volume de amostra armazenado ser relativamente alto) pode ter acarretado a variação inesperada em alguns resultados, como é observado para a amostra armazenada por 11 meses em tonel de madeira, que apresentou uma concentração de carbamato de etila inferior àquela observada para a amostra anteriormente coletada (armazenada por 10 meses).

Diversos trabalhos realizados com aguardentes têm mostrado que muitas das amostras analisadas apresentaram concentrações de CE acima do limite máximo estabelecido pela legislação $(150 \mu \mathrm{g}$ $\left.\mathrm{L}^{-1}\right) .{ }^{10,19,20}$ Nota-se que, mesmo havendo um aumento na concentração de $\mathrm{CE}$ ao longo do período de estocagem da cachaça, a concentração deste contaminante esteve inferior ao limite máximo estabelecido pelo MAPA $\left(150 \mu \mathrm{g} \mathrm{L}^{-1}\right)$.

Por meio do teste de variância aplicado às médias de cada procedimento de estocagem da cachaça, constatou-se diferença significativa na concentração de CE para ambos os procedimentos. Para ambos, foi observado um aumento na concentração de CE, sendo este aumento mais expressivo para a cachaça armazenada em recipiente de vidro. Apesar disso, pode-se notar uma tendência de estabilização na concentração do CE para a cachaça proveniente do recipiente de vidro, enquanto que para a cachaça envelhecida o aumento na concentração de $\mathrm{CE}$ tendeu a ser progressivo durante o período de estocagem da bebida. Estes resultados estão condizentes com aqueles obtidos por Madrera e Valles, ${ }^{12}$ os quais apontaram um aumento progressivo na concentração de CE em cidras submetidas a diferentes níveis de maturação.

Andrade Sobrinho et al. ${ }^{4}$ simularam o tempo de "descanso" da cachaça, como também o tempo de permanência da bebida na prateleira, antes de seu consumo, e constataram que a concentração de CE após 36 meses de estocagem da cachaça variou de $-5,3$ a $6,7 \%$, sugerindo a estabilidade na concentração deste composto após a sua formação.

Apesar de mencionar que, aparentemente, não foi encontrada associação entre a concentração de CE e a coloração de cachaças (que, segundo os autores, a coloração amarelada da bebida indicaria o armazenamento em barris de madeira) analisadas de diferentes destilarias da Paraíba, Nóbrega et al. ${ }^{21}$ citam que em 2 das destilarias estudadas a concentração de CE encontrada para a cachaça branca foi maior do que para a cachaça amarelada. Isso mostra que, possivelmente, este contaminante pode ser formado, não somente quando a bebida é mantida estocada em recipientes que não são de madeira, mas também quando há a estocagem da cachaça em barris e toneis de diferentes espécies de madeiras.

Acredita-se que a formação do CE durante o período de armazenamento da cachaça aconteça de maneira gradativa, por meio da reação entre o etanol e a ureia formada por meio da degradação de precursores nitrogenados, intrínsecos ao processo de produção da bebida, sendo os principais deles os aminoácidos arginina, ornitina e citrulina. Além destes, outros compostos nitrogenados também têm sido estudados como possíveis precursores para a formação do carbamato de etila antes e após o processo de destilação, como é o caso do fosfato de carbamila e do íon cianeto. ${ }^{22,23}$

Estudos de Hasnip et al., ${ }^{24}$ avaliando a influência do tempo de estocagem e da temperatura na concentração de carbamato de etila e seus precursores em vinhos, mostraram uma diminuição nas concentrações de ureia e citrulina, enquanto houve um aumento na concentração de CE. Este fato evidencia a participação destes compostos na formação do CE, possibilitando a análise cinética da formação desta substância associada à concentração de etanol, ureia e citrulina em vinhos.

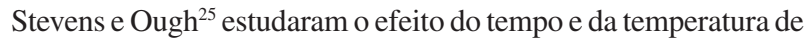
estocagem de vinhos, durante um período de 2 anos, na formação do CE. Avaliaram a formação do CE por meio da reação do etanol com ureia e com citrulina, sob diferentes temperaturas de estocagem, em garrafas de $125 \mathrm{~mL}$. Os resultados mostraram que a concentração de ureia e de citrulina e a temperatura de estocagem do vinho influenciaram de maneira positiva a formação de CE durante o período de estocagem da bebida.

\section{CONCLUSÕES}

O método cromatográfico proposto mostrou-se eficiente para a determinação do carbamato de etila em cachaça, podendo ser uma alternativa ao método considerado como oficial até o momento.

$\mathrm{O}$ armazenamento da bebida, tanto em tonel de carvalho quanto em recipiente de vidro, influenciou na formação do carbamato de etila, proporcionando um aumento significativo na concentração deste contaminante. Este fato contribui para os estudos de controle e acompanhamento da qualidade da cachaça, uma vez que sugere que a formação do carbamato de etila ocorre de maneira significativa durante o período de armazenamento da bebida, dando suporte para posteriores estudos com relação à origem e formação deste composto na mesma. 


\section{AGRADECIMENTOS}

Ao Programa REUNI/CAPES, ao CNPq e à FAPEMIG pelo suporte financeiro. Ao produtor da Cachaça Artesanal João Mendes pelo fornecimento das amostras.

\section{REFERÊNCIAS}

1. Cardoso, M. G. Em Produção de Aguardente de Cana; Cardoso, M. G., ed.; Editora UFLA: Lavras, 2006, 2a ed., p. 203-232.

2. Bruno, S. N. F.; Vaitsman, D. S.; Kunigami, C. N.; Brasil, M. G.; Food Chem. 2007, 104, 1345.

3. Labanca, R. A.; Glória, M. B. A.; Afonso, R. J. C. F.; Quim. Nova 2008, $31,1860$.

4. Andrade Sobrinho, L. G.; Cappelini, L. T. D.; Silva, A. A.; Galinaro, C. A.; Bushviser, S. F.; Cardoso, D. R.; Franco, D. W.; Quim. Nova 2009, $32,116$.

5. BRASIL, Ministério da Agricultura, Pecuária e Abastecimento (MAPA); Instrução Normativa n.13, de 29 de junho de 2005.

6. Weber, J. V.; Sharypov, V. I.; Environ. Chem. Lett. 2008, 7, 233.

7. Andrade-Sobrinho, L. G.; Boscolo, M.; Lima-Neto, B. S.; Franco, D. W.; Quim. Nova 2002, 25, 1074.

8. Polastro, L. R.; Boso, L. M.; Andrade-Sobrinho, L. G.; Lima-Neto, B. S.; Franco, D. W.; Ciênc. Tecnol. Aliment. 2001, 21, 78.

9. Iida, Y.; Suganuma, Y.; Matsumoto, K.; Satoh, I.; Anal. Sci. 2006, 22, 173.

10. Masson, J.; Tese de Doutorado, Universidade Federal de Lavras, Brasil, 2009.
11. Abreu, S. M.; Alves, A.; Oliveira, B.; Herbert, P.; Anal. Bioanal. Chem. 2005, 382, 498.

12. Madrera, R. R.; Valles, B. S.; Food Control 2009, 20, 139.

13. Herbert, P.; Santos, L.; Bastos, M.; Barros, P.; Alves, A.; J. Food Sci. 2002, 67, 1616 .

14. Park, S.; Kim, C. T.; Lee, J.; Jhee, O. H.; Om, A. S.; Kang, J. S.; Moon, T. W.; Food Control 2007, 18, 975.

15. Harris, D. C.; Análise Química Quantitativa, $7^{\mathrm{a}}$ ed., trad. Jairo Bordinhão [et al.], LTC: Rio de Janeiro, 2008.

16. Machado, A. M. R.; Tese de Doutorado, Universidade Federal de Lavras, Brasil, 2010.

17. Ferreira, D. F.; SISVAR, versão 4.6, bluild 61; Universidade Federal de Lavras, Lavras, 2003.

18. Aresta, M.; Boscolo, M.; Franco, D. W.; J. Agric. Food Chem. 2001, 49, 2819.

19. Barcelos, L. V. F.; Cardoso, M. G.; Vilela, F. J.; Anjos, J. P.; Quim. Nova 2007, 30, 1009 .

20. Zacaroni, L. M.; Dissertação de Mestrado, Universidade Federal de Lavras, Brasil, 2009.

21. Nóbrega, I. C. C.; Pereira, J. A. P.; Paiva, J. E.; Lachenmeier, D. W.; Food Chem. 2009, 117, 693.

22. Cook, R.; Mccaig, N.; Mcmillan, J. M. B.; Lumsden, W. B.; J. Inst. Brew. 1990, 96, 233.

23. Lawrence, J. F.; Page, B. D.; Conacher, H. B. S. ; Advice Environment Science Technology 1990, 23, 457.

24. Hasnip, S.; Caputi, A.; Crews, C.; Brereton, P.; Food Addit. Contam., Part A 2004, 21, 1155.

25. Stevens, D. F.; Ough, C. S.; Am. J. Enol. Vitic. 1993, 44, 309. 